\title{
(De)generative Art: Rules-transgressing algorithms
}

\author{
Dario Srbic \\ Royal College of Art \\ 1 Hester Road \\ London SW11 4AN, UK \\ dario.srbic@network.rca.ac.uk
}

\section{FROM RATIONAL TO IRRATIONAL ALGORITHMS}

While Descartes famous phrase "I think therefore I am" from 1637 was asking what does it mean to be human (Descartes 2001), this paper and demonstration explore a possibility of embodying desire, understood as a transgression of a law (Bataille 1986), into algorithms and letting them express those transgressions as artworks. Transgression, etymologically a "cross-over", implies in Bataille a move from ordered, rational state into to unordered, irrational realm. If we think in traditional computing terms of the algorithm as a set of pre-defined rules, then it is hard to imagine any possibility of those rules being broken, without inherently breaking the algorithm. Only with the advent of artificial intelligence, notably different forms of neural networks, the notion of hard set of rules with a precisely defined sequence to be followed becomes softer, with algorithms' ability to adopt and change the rules governing the solution to a given problem. Here, the knowledge as a recognition is being formed. It implies a passage from "not-recognising" to "recognising" and hence stresses the importance of the process. Depending on the complexity of the network, this process can be highly "irrational", developing an illogical solution never imagined by a researcher or programmer. By recognising patters, algorithms gain a sense of knowledge and agency, developing their own rules, instead of merely following them, embodying an ability that is truly their own. This is how most generative art is being produced, with algorithms creating work that possesses idiosyncrasy of a human artist, and in certain cases indistinguishable from a human production. At this stage in the demonstration, the neural networks are trained to classify different biometric measurements from my body to recognise different feelings. While in possession of the knowledge, algorithms still lack notions of ethics and excess, without which transgression is not possible.

\section{NEURAL NETWORK ETHICS}

The current design of artificial intelligence supports and nourishes the power of the technological, which already operates on a planetary scale, forming a technosphere (Klose 2016), the mobilisation and hybridisation of energy, material, and environments, that create ecological catastrophes and force sentient beings to take a backseat to the information, big data and deep learning. Optimised for exploitation and profit maximisation, neural networks lack the sense of the consequences of their actions, notably when they result in norm violations and harming another sentient being. Mechanisms like pain or pleasure, embodied in our own entanglement of being, knowledge and ethics, need to find a way into artificial intelligence, in order to move algorithms from mere recognition to another form of knowledge, which possesses an ethical dimension and brings along the care of oneself and others (Foucault 2011). It is evidently not enough to implement Isaac Asimov's laws (Asimov 1956) of not hurting another human being in form of traditional algorithmic constraints or again as a set of rules. The ethical moment needs to be embodied in a form of post-action, considering an "affective residue" of past feelings, that can serve as a predictor of emotional outcomes of future behaviours (Matarazzo \& Baldassarre 2015). If irrational algorithms could detect those emotions and know about them in form of recognition, in this step the care for those emotions is a part of problem-solving process, receiving its own weight in network neurones. Only with this knowledge, algorithms can sense the outcome their actions have on sentient beings, move away from relentless forward progress and incorporate lateral development that takes into account and care about living organisms. While it is still not possible to objectively detect and accurately measure nociception or pain via physiological markers only (Cowen et al. 2015), by combining subjective 
reporting of pain and biometric data, the neural network used in the demonstration can be trained to incorporate those measurements while searching for an optimal solution, and thus forming a rudimentary ethical framework. By including physiological data in the training feedback, the algorithms have a chance to learn of their impact on sentient beings, and are making a crucial step towards transgression and (de)generative art.

\section{EXCESS IN AN ALGORITHMIC ART}

In contemporary realm (as seen through the lens of post-structuralism), transgression plays an important role in defining what is "appropriate" and "inappropriate". This continuous oscillation between those two modes is crucial in constructing of self, the other, knowledge and power (Foucault 1990). In addition to this boundary or definition of limit, transgression as "excess" challenges the rational and involves pleasure when crossing from the ordered into irrational state. The pleasure in the case of transgression is usually accompanied by guilt, embarrassment and shame. Having algorithms that can recognise, classify and even act upon our feelings, allows to look at possible scenarios for transgression to take place. Here, a way stronger bond and exchange between the body and algorithms is need to convey the complexity of different feelings and their interplay when dealing with transgression. Can algorithms practice excess by deviating from optimum weighting between parameters and giving excess importance to pleasure and satisfaction? Just like the robot in the demonstration serves as a prosthesis to expressing feelings and miming style, so can the body in the demonstration provide a cognitive prosthesis by provoking a transgression and scrambling of trained neural networks. Through data, that might under circumstances signal at the same time pleasure and pain, satisfaction and shame, algorithms might learn to hurt in order to please, crossing the limit and exploring the boundary and exceeding what they were meant to learn. At this moment, a possibility of (de)generative, algorithmic art emerges, that does not only create pleasing or unpleasing images, but embodies feelings from its somatic, sentient prosthesis. Hidden in a neural network, a behavioural pattern may lie latent, waiting to be triggered only in special circumstances, when the network senses the opportunity to transgress and negotiate new borders. In this demonstration, the body and algorithms are not only expressing feelings and possible excess, but also exist on that boundary, crossing into each other realms, not merely representing but performing transgression.

\section{REFERENCES}

Asimov, I. (1950) Runaround. In I, Robot. Doubleday, New York.

Bataille, G. (1986) Erotism: Death and Sensuality. University of Minnesota Press, Minneapolis.

Cowen, R., Stasiowska, M. K., Laycock, H., and Bantel, C. (2015) Assessing pain objectively: the use of physiological markers. Anaesthesia, 70, 828-847.

Descartes, R. (2011) Discourse on Method, Optics, Geometry, and Meteorology. Hackett Publishing, Indianapolis.

Foucault, M. (2011) The Courage of Truth. Palgrave Macmillan UK, London.

Foucault, M. (1990) The Use of Pleasure. Vintage Books, New York.

Klose, A. (2016) Technosphere Magazine: Container Love, and Fear. https://technospheremagazine.hkw.de/p/c5843f70-944d-11e6-b1279b6412115574 (retrieved 12 February 2018).

Matarazzo, O. and Baldassarre, I. (2015) Are Emotions Reliable Predictors of Future Behavior?. In Bassis, S. et al. (eds.), Recent Advances of Neural Networks Models and Applications. Springer International Publishing, Cham. 